\title{
Impact of ALDH2 Gene Polymorphism on Coronary Artery lesions in Ethnic Hakka Population with Acute Myocardial Infarction
}

\section{Caiyan Gan}

Meizhou People's Hospital

\section{Sudong Liu}

Meizhou People's Hospital

\section{Xuemin Guo}

Meizhou People's Hospital

\section{Ruiqiang Weng}

Meizhou People's Hospital

Xiaodong Gu

Meizhou People's Hospital

Zhixiong Zhong ( $\square$ zhongzhixiong@mzrmyy.com )

Meizhou People's Hospital https://orcid.org/0000-0002-3200-3105

\section{Research article}

Keywords: ALDH2 mutated allele, coronary artery lesions, acute myocardial infarction

Posted Date: June 12th, 2020

DOl: https://doi.org/10.21203/rs.3.rs-34691/v1

License: (1) This work is licensed under a Creative Commons Attribution 4.0 International License. Read Full License 


\section{Abstract}

Background: Aldehyde dehydrogenase 2 (ALDH2) contributes to converting acetaldehyde into acetate, thus plays a key role in the ethanol metabolism and oxidation of acetaldehyde. The aim of this study is to investigate the impact of Aldehyde dehydrogenase 2 (ALDH2) gene polymorphism on coronary artery lesions in ethnic Hakka patients with acute myocardial infarction (AMI). Methods: A total of 312 patients (male $=241$, female $=71$ ) with acute myocardial infarction (AMI) suffered $\geq 50 \%$ stenosis of at least one major coronary artery were recruited into the study. Lesions of AMI patients carried different ALDH2 genotypes were analyzed. Results: The incidence of multivessel lesions was higher in the ALDH2 mutation group (90.6\%) than the ALDH2 wild group (80.3\%) with reaching statistical significance ( $p=$ 0.009). Compare to the single lesion group, multivessel lesions group were more likely to be lower DBP (77.3 \pm 14.1 versus $86.4 \pm 13.8, p=0.004)$, older $(66.1 \pm 12.2$ versus $59 \pm 12.1, p=0.009$ ), have hypertension ( $55.7 \%$ versus $30.8 \%, p=0.023$ ) in the ALDH2 wild patients. In the ALDH2 mutation patients, multivessel lesions group also tend to be elderly $(65.9 \pm 11.3$ versus $58.5 \pm 11.3, p=0.011)$. In the addition, the multivessel lesions group presented higher levels of the TG and lower levels of the LDL-C than the single lesion group, but it is no statistical differences. The logistics regression showed that age and ALDH2 mutation were vulnerable to the multivessel lesions the ethnic Hakka population with AMI. Conclusions: The AMI patients in ethnic Hakka population carrying mutated ALDH2 allele was vulnerable to multiple coronary artery lesions. ALDH2 allele was associated with multiple coronary artery lesions in AMI patients among ethnic Hakka population. Key words: ALDH2 mutated allele, coronary artery lesions, acute myocardial infarction

\section{Background}

Acute myocardial infarction (AMI) is a clinically critical disease and it leads to a high risk of mortality and morbidity worldwide especially in the developed countries. Approximately 550,000 first episodes and 200,000 recurrent episodes of acute myocardial infarction occur annually[1,2]. In China, AMI has been a common disease that poses a great threat to human health and socioeconomic burden due to the changes of economy situation and life styles[3]. AMI is caused by an occlusive thrombus at the site of a ruptured coronary plaque that is the most severe type of coronary artery disease (CAD). It is divided into ST-segment elevation myocardial infarction (STEMI) and non-ST-segment elevation myocardial infarction (NSTEMI) based on the presence or absence of ST-segment elevation on the electrocardiogram[4]. It is well known that multiple factors such as diabetes, dyslipidemia, hypertension, smoking, obesity, metabolic syndrome, age and air pollution are closely associated with the risk of AMI[5-9]. Studies showed that up to $50 \%$ of the AMI patients suffered multivessel lesions as examined by coronary artery angiographic $[10,11]$. Multivessel lesions patients can have more serious clinical manifestations, poorer prognosis and a 2-fold increase in 1-year mortality than single lesion [12]. The assessment of pexelizumab in acute myocardial infarction (APEX-AMI) study showed that the all-cause mortality rate of patients with single-vessel lesion and multivessel lesions AMI patients was $3.1 \%$ and $6.3 \%$, respectively, 
and multivessel lesions was an independent risk factor for predicting death and reinfarction in patients[13].

Aldehyde dehydrogenase 2 (ALDH2) contributes to converting acetaldehyde into acetate, thus plays a key role in the ethanol metabolism and oxidation of acetaldehyde[14]. ALDH2 is also the key enzyme for nitroglycerin metabolism. Carriers of the $\mathrm{ALDH} 2^{*} 2$ genetype is involved in the transformation from nitroglycerin to nitric oxide that plays a crucial role in nitroglycerin tolerance in Asian patients with CAD [15]. The ALDH2 gene consisted of 13 exons on chromosome 12. There are three genotypes named wild type $\left({ }^{*} 1 /{ }^{*} 1\right.$, homozygote), mutated type $\left({ }^{*} 1 /^{*} 2\right.$, heterozygote and ${ }^{*} 2 /^{*} 2$, homozygote) and two ALDH2 alleles (ALDH2 ${ }^{*} 1$ and $A L D H 2{ }^{*} 2$ ) owing to a single nucleotide polymorphism (rs671) leads to the transition of guanine $(G)$ to adenine $(A)$, at last, an amino acid change at position 504 from glutamate to lysine in the ALDH2 protein[15-17]. The research showed that ALDH2 heterozygous individuals (ALDH2 ${ }^{*} 1 /{ }^{*} 2$ ) had only $6.25 \%$ of the enzymatic activity compared to the ALDH2 homozygous individuals (ALDH2* $1 /{ }^{*} 1$ ), while ALDH2 ${ }^{*} 2 /^{*} 2$ basically eliminated its dehydrogenase activity[18]. The variant of ALDH2 gene Glu504Lys is frequent in East Asian population with a prevalence of $30-50 \%[15]$.

At present, the relationship of ALDH2 gene polymorphism and coronary artery lesions in AMI patients of ethnic Hakka population remains unclear. In this study, we aimed to investigate the impact of ALDH2 gene polymorphism on coronary artery lesions in AMI patients among Hakka population.

\section{Methods}

\section{Subjects}

The study subjects were choosed from patients who attended the cardiology department of Meizhou People's Hospital and diagnosed with AMI during August 2016 and May 2019. A total of 312 patients with AMI suffered $\geq 50 \%$ stenosis of at least one major coronary artery were recruited into the study. All subjects were Hakka ethnic population residing in the Meizhou city. Definition of diseased vessel: left main disease and 3 subepicardial coronary artery, left anterior descending, left circumflex, right coronary stenosis $\geq 50 \%$ by coronary angiography were defined as coronary lesions. Only one vessel involvement was defined as a single vessel lesion, and more than single vessel lesion was identified as multivessel lesions. This study was performed according to the ethical guidelines of the Helsinki Declaration and approved by the Human Ethics Committees of the Meizhou People's Hospital (Huangtang Hospital), Meizhou Academy of Medical Sciences, Meizhou Hospital Affiliated to Sun Yat-sen University, Guangdong Province, China. All participants had signed the informed consent before the study.

\section{DNA extraction and genotyping}

Two milliliters of peripheral venous blood sample was collected in vacuum tubes containing EDTA. Genomic DNA was extracted from the blood of subjects using the TIANamp Blood DNA Kit (Tiangen, Beijing, China) following the manufacturer's instructions, and DNA concentration was evaluated by using a Nanodrop $2000^{\text {TM }}$ Spectrophotometer (Thermo Fisher Scientific, Waltham, MA). Genotyping of ALDH2 
Glu504Lys (rs671) polymorphisms was performed by polymerase chain reaction (PCR) amplification, and using a commercial detection kit (BaiO Technology Co, Ltd., Shanghai, China) was subjected to hybridization reaction. The $25 \mu \mathrm{L}$ polymerase chain reaction mixture contained 20 120 ng DNA template and the recommended amounts of primers, Taq DNA polymerase and dNTPs. The PCR cycling conditions was performed according to the protocol: $50^{\circ} \mathrm{C}$ for $5 \mathrm{~min}$, predenaturation at $94^{\circ} \mathrm{C}$ for $5 \mathrm{~min}$, followed by 35 cycles at $94^{\circ} \mathrm{C}$ for $25 \mathrm{~s}, 60^{\circ} \mathrm{C}$ for $25 \mathrm{~s}$ and $72^{\circ} \mathrm{C}$ for $30 \mathrm{~s}$, and final extension at $72^{\circ} \mathrm{C}$ for $5 \mathrm{~min}$. The 10 $\mu \mathrm{L}$ amplifcation products were then dispensed into $190 \mu \mathrm{L}$ of hybridization buffer to carry out the hybridization reaction. The genotypes of ALDH2 were visualized by using the BaiO Array Doctor Version 2.0 software (BaiO Technology Co, Ltd., Shanghai, China), according to the manufacturer's instructions.

\section{Statistical analysis}

Statistical Packagefor the Social Sciences (SPSS), version 21.0 was used for data analysis. The data are presented as the means \pm SD. Chi-square test and Student's $t$-test were used to compare the wild and mutated genetype. Multivariate logistic regression analyses were used to determine the predictors of multivessel lesions in patients with different genotypes. A value of $p<0.05$ was considered as statistically significant.

\section{Results}

\section{ALDH2 genotypes and AMI patients with coronary artery lesions}

A total of 312 subjects were enrolled, including 132 AMI patients with ALDH2 wild genotype $\left({ }^{*} 1 /{ }^{*} 1\right)$ and 180 AMI patients with ALDH2 mutation $\left({ }^{*} 1 /{ }^{*} 2\right.$ and $\left.{ }^{*} 2 /{ }^{*} 2\right)$, respectively. Demographic characteristics for all subjects were shown in Table 1. There were 241 males and 71 females. The mean age of these patients was $65 \pm 11.9$. Among them, $46.2 \%$ smoked cigarettes, $5.4 \%$ alcohol drinking, $49 \%$ hypertension, $29.2 \%$ diabetes, $86.2 \%$ multivessel lesions, and $93.3 \%$ patients had suffered from LAD lesion. There were no significant differences in age, gender, SBP, DBP, smoking, hypertension, CREA, ALT, number of diseased vessels, location lesion, TG, TC, HDL-C, LDL-C between wild type group and mutated group. However, percent of multivessel lesions was significantly difference in ALDH2 mutated genotype group than in ALDH2 wild group $(90.6 \%$ versus $80.3 \%, p=0.009)$. In addition, patients with AMI with wild-type ALDH2 $\left({ }^{*} 1 /^{*} 1\right)$ showed significantly higher alcohol consumption than did those with ${ }^{*} 1 /^{*} 2$ and ${ }^{*} 2 /^{*} 2$ genotypes $(2.2 \%$ versus $9.8 \%, \mathrm{P}=0.003)$. 
Table 1

Characteristics of ALDH2 Wild and Mutated Subjects

\begin{tabular}{|c|c|c|c|c|}
\hline Variable & $\begin{array}{l}\text { Total } \\
(n=312)\end{array}$ & $\begin{array}{l}{ }^{*} 1{ }^{*} 1 \\
(n=132)\end{array}$ & $\begin{array}{l}{ }^{*} 1 /^{*} 2+{ }^{*} 2 /^{*} 2 \\
(n=180)\end{array}$ & $P$ value \\
\hline Age(years) & $65 \pm 11.9$ & $64.7 \pm 12.5$ & $65.2 \pm 11.5$ & 0.679 \\
\hline Gender(Male/Female) & $241 / 71$ & $104 / 28$ & $137 / 43$ & 0.577 \\
\hline $\mathrm{SBP}(\mathrm{mmHg})$ & $131.2 \pm 24$ & $132.8 \pm 26$ & $130.1 \pm 22.4$ & 0.325 \\
\hline $\mathrm{DBP}(\mathrm{mmHg})$ & $77.7 \pm 14$ & $79.1 \pm 14.4$ & $76.7 \pm 13.6$ & 0.128 \\
\hline Alcohol(n,\%) & $17(5.4 \%)$ & 13(9.8\%) & $4(2.2 \%)^{\star \star}$ & 0.003 \\
\hline Smoking(n,\%) & $144(46.2 \%)$ & $64(48.5 \%)$ & $80(44.4 \%)$ & 0.479 \\
\hline Hypertension(n,\%) & $153(49 \%)$ & $67(50.8 \%)$ & $86(47.8 \%)$ & 0.603 \\
\hline Diabetes(n,\%) & $91(29.2 \%)$ & $36(27.3 \%)$ & $55(30.6 \%)$ & 0.529 \\
\hline $\operatorname{CREA}(\mu \mathrm{mol} / \mathrm{L})$ & $109.2 \pm 43.8$ & $109.2 \pm 43.4$ & $109.2 \pm 44.2$ & 0.996 \\
\hline $\mathrm{ALT}(\mathrm{U} / \mathrm{L})$ & $53.9 \pm 42.8$ & $55.2 \pm 45.5$ & $53 \pm 40.8$ & 0.661 \\
\hline Number of diseased vessels & $2.6 \pm 0.9$ & $2.5 \pm 1.0$ & $2.6 \pm 0.8$ & 0.103 \\
\hline \multicolumn{5}{|l|}{ Location lesion [n(\%)] } \\
\hline LM & $51(16.3 \%)$ & $23(17.4 \%)$ & $28(15.6 \%)$ & 0.659 \\
\hline LAD & 291(93.3\%) & $122(92.4 \%)$ & $169(93.9 \%)$ & 0.610 \\
\hline LCX & $214(68.6 \%)$ & $83(62.9 \%)$ & $131(72.8 \%)$ & 0.063 \\
\hline RCA & $241(77.2 \%)$ & $97(73.5 \%)$ & $144(80 \%)$ & 0.175 \\
\hline \multicolumn{5}{|l|}{ Laboratory parameters } \\
\hline $\mathrm{TG}\left(\mathrm{mmol} \cdot \mathrm{L}^{-1}\right)$ & $1.98 \pm 1.47$ & $2.06 \pm 1.42$ & $1.92 \pm 1.51$ & 0.402 \\
\hline $\mathrm{TC}\left(\mathrm{mmol} \cdot \mathrm{L}^{-1}\right)$ & $5.21 \pm 1.37$ & $5.24 \pm 1.37$ & $5.19 \pm 1.38$ & 0.793 \\
\hline $\mathrm{HDL}-\mathrm{C}\left(\mathrm{mmol} \cdot \mathrm{L}^{-1}\right)$ & $1.18 \pm 0.28$ & $1.19 \pm 0.28$ & $1.18 \pm 0.28$ & 0.700 \\
\hline $\mathrm{LDL}-\mathrm{C}\left(\mathrm{mmol} \cdot \mathrm{L}^{-1}\right)$ & $3.11 \pm 1.01$ & $3.16 \pm 1.06$ & $3.08 \pm 0.97$ & 0.496 \\
\hline Multivessel Lesion(n,\%) & $269(86.2 \%)$ & $106(80.3 \%)$ & $163(90.6 \%) \star \star$ & 0.009 \\
\hline \multicolumn{5}{|c|}{$\begin{array}{l}\text { Data are presented as mean } \pm \text { SD. }{ }^{*} p<0.05,{ }^{* *} p<0.01 \text {. SBP, systolic blood pressure; DBP, diastolic } \\
\text { blood pressure; CREA, creatinine; } A L T \text {, alanine transaminase; } L M \text {, left main disease; LAD, left anterior } \\
\text { descending artery; LCX, left circumflex; RCA, right coronary artery; TG, triglyceride; TC, total cholesterol; } \\
\text { HDL-C, high density lipoprotein cholesterol; LDL-C, low density lipoprotein cholesterol. }\end{array}$} \\
\hline
\end{tabular}


Patients with ALDH2 wild genotype vessel lesion characteristics are shown in Table 2. In ALDH2 wild genotype AMI patients, we divided into the single lesion group $(n=26)$ and the multivessel lesions group $(n=106)$. Compared with the single lesion group, the multivessel lesions group were more older in age (66.1 \pm 12.2 versus $59 \pm 12.1, p=0.009)$, had higher CREA levels and a significant differences in the location lesion ( $L M, L A D, L C X, R C A, p<0.01)$, DBP, smoking, hypertension, $T G$, number of diseased vessels between the two groups. 
Table 2

Effect of Multivessel Lesions on Patients with ALDH2 Wild Genotype

\begin{tabular}{|c|c|c|c|c|}
\hline Variable & $\begin{array}{l}\text { Total } \\
(n=132)\end{array}$ & $\begin{array}{l}\text { Single Lesion } \\
(n=26)\end{array}$ & $\begin{array}{l}\text { Multivessel Lesions } \\
(n=106)\end{array}$ & $P$ value \\
\hline Age(years) & $64.7 \pm 12.5$ & $59 \pm 12.1$ & $66.1 \pm 12.2^{\star \star}$ & 0.009 \\
\hline Gender(Male/Female) & $104 / 28$ & $23 / 3$ & $81 / 25$ & 0.178 \\
\hline $\mathrm{SBP}(\mathrm{mmHg})$ & $132.8 \pm 26$ & $140 \pm 24.8$ & $131 \pm 26.1$ & 0.114 \\
\hline $\mathrm{DBP}(\mathrm{mmHg})$ & $79.1 \pm 14.4$ & $86.4 \pm 13.8$ & $77.3 \pm 14.1^{\star *}$ & 0.004 \\
\hline Alcohol(n,\%) & $13(9.8 \%)$ & $5(19.2 \%)$ & $8(7.5 \%)$ & 0.073 \\
\hline Smoking(n,\%) & $64(48.5 \%)$ & $18(69.2 \%)$ & $46(43.4 \%)^{\star \star}$ & 0.018 \\
\hline Hypertension(n,\%) & $67(50.8 \%)$ & $8(30.8 \%)$ & $59(55.7 \%)^{\star \star}$ & 0.023 \\
\hline Diabetes(n,\%) & $36(27.3 \%)$ & $5(19.2 \%)$ & $31(29.2 \%)$ & 0.304 \\
\hline $\operatorname{CREA}(\mu \mathrm{mol} / \mathrm{L})$ & $109.2 \pm 43.4$ & $98.8 \pm 15.7$ & $111.7 \pm 47.5$ & 0.175 \\
\hline $\operatorname{ALT}(\mathrm{U} / \mathrm{L})$ & $55.2 \pm 45.5$ & $63 \pm 55.2$ & $53.2 \pm 42.9$ & 0.328 \\
\hline $\begin{array}{l}\text { Number of } \\
\text { diseased vessels }\end{array}$ & $2.5 \pm 1$ & $1 \pm 0$ & $2.8 \pm 0.7^{\star \star}$ & 0 \\
\hline \multicolumn{5}{|l|}{ Location lesion [n(\%)] } \\
\hline LM & $23(17.4 \%)$ & $0(0)$ & $23(21.7 \%)^{\star \star}$ & 0.009 \\
\hline LAD & $122(92.4 \%)$ & $20(76.9 \%)$ & $102(96.2 \%)^{* *}$ & 0.001 \\
\hline LCX & $83(62.9 \%)$ & $3(11.5 \%)$ & $80(75.5 \%)^{\star \star}$ & 0 \\
\hline RCA & 97 (73.5\%) & $3(11.5 \%)$ & $94(88.7 \%)^{\star \star}$ & 0 \\
\hline \multicolumn{5}{|l|}{ Laboratory parameters } \\
\hline $\mathrm{TG}\left(\mathrm{mmol} \cdot \mathrm{L}^{-1}\right)$ & $2.06 \pm 1.42$ & $2.56 \pm 2.06$ & $1.94 \pm 1.20^{*}$ & 0.049 \\
\hline $\mathrm{TC}\left(\mathrm{mmol} \cdot \mathrm{L}^{-1}\right)$ & $5.24 \pm 1.37$ & $4.93 \pm 1.15$ & $5.31 \pm 1.41$ & 0.201 \\
\hline $\mathrm{LDL}-\mathrm{C}\left(\mathrm{mmol} \cdot \mathrm{L}^{-1}\right)$ & $3.16 \pm 1.06$ & $2.85 \pm 0.91$ & $3.23 \pm 1.08$ & 0.096 \\
\hline
\end{tabular}

Data are presented as mean $\pm S D$. ${ }^{*} p<0.05$, ${ }^{*} p<0.01$ (Multivessel Lesions vs Single Lesion). SBP, systolic blood pressure; DBP, diastolic blood pressure; CREA, creatinine ; ALT, alanine transaminase; $L M$, left main disease; $L A D$, left anterior descending artery; LCX, left circumflex; RCA, right coronary artery; TG, triglyceride; TC, total cholesterol; HDL-C, high density lipoprotein cholesterol; LDL-C, low density lipoprotein cholesterol. 


\begin{tabular}{|c|c|c|c|c|}
\hline Variable & $\begin{array}{l}\text { Total } \\
(n=132)\end{array}$ & $\begin{array}{l}\text { Single Lesion } \\
(n=26)\end{array}$ & $\begin{array}{l}\text { Multivessel Lesions } \\
(n=106)\end{array}$ & $P$ value \\
\hline $\mathrm{HDL}-\mathrm{C}\left(\mathrm{mmol} \cdot \mathrm{L}^{-1}\right)$ & $1.19 \pm 0.28$ & $1.19 \pm 0.34$ & $1.19 \pm 0.26$ & 0.966 \\
\hline \multicolumn{5}{|c|}{$\begin{array}{l}\text { Data are presented as mean } \pm \text { SD. }{ }^{*} p<0.05, * * p<0.01 \text { (Multivessel Lesions vs Single Lesion). SBP, } \\
\text { systolic blood pressure; DBP, diastolic blood pressure; CREA, creatinine; ALT, alanine transaminase; } \\
\text { LM, left main disease; LAD, left anterior descending artery; LCX, left circumflex; RCA, right coronary } \\
\text { artery; TG, triglyceride; TC, total cholesterol; HDL-C, high density lipoprotein cholesterol; LDL-C, low } \\
\text { density lipoprotein cholesterol. }\end{array}$} \\
\hline
\end{tabular}

\section{Patients with ALDH2 mutated Genotype and multivessel Lesions}

Patients with ALDH2 mutated genotype vessel Lesion characteristics are shown in Table 3. In this cohort of 180 patients with mutated genotype ( 137 male, 43 female) were no significant differences between the single lesion and the multivessel lesions group. For mutated genotype patients, the average age were significantly difference between single lesion and multivessel lesions (65.9 versus $58.5, p=0.011)$. More than half of the patients in multivessel lesions group had LAD, LCX, RCA (95.7\%, 77.9\%, 88.3\%)location lesion is significant differences $(p<0.01)$ compare to the single lesion group. The number of diseased vessels were significantly higher in the multivessel lesions group $(2.8 \pm 0.7)$ than in the single lesion group $(1 \pm 0)(p<0.01)$. 
Table 3

Effect of Multivessel Lesions on Patients with ALDH2 Mutated Genotype

\begin{tabular}{|c|c|c|c|c|}
\hline Variable & $\begin{array}{l}\text { Total } \\
(n=180)\end{array}$ & $\begin{array}{l}\text { Single Lesion } \\
(n=17)\end{array}$ & $\begin{array}{l}\text { Multivessel Lesions } \\
(n=163)\end{array}$ & $P$ value \\
\hline Age(years) & $65.2 \pm 11.5$ & $58.5 \pm 11.3$ & $65.9 \pm 11.3^{*}$ & 0.011 \\
\hline Gender(Male/Female) & $137 / 43$ & $14 / 3$ & $123 / 40$ & 0.526 \\
\hline $\mathrm{SBP}(\mathrm{mmHg})$ & $130.1 \pm 22.4$ & $129.7 \pm 17.4$ & $130.1 \pm 22.9$ & 0.944 \\
\hline $\mathrm{DBP}(\mathrm{mmHg})$ & $76.7 \pm 13.6$ & $77.2 \pm 10.5$ & $76.6 \pm 13.9$ & 0.876 \\
\hline Alcohol(n,\%) & $4(2.2 \%)$ & $1(5.9 \%)$ & $3(1.8 \%)$ & 0.282 \\
\hline Smoking $(\mathrm{n}, \%)$ & $80(44.4 \%)$ & $11(64.7 \%)$ & $69(42.3 \%)$ & 0.077 \\
\hline Hypertension(n,\%) & $86(47.8 \%)$ & $9(52.9 \%)$ & $77(47.2 \%)$ & 0.654 \\
\hline Diabetes $(n, \%)$ & $55(30.6 \%)$ & $5(29.4 \%)$ & $50(30.7 \%)$ & 0.914 \\
\hline CREA $(\mu \mathrm{mol} / \mathrm{L})$ & $109.2 \pm 44.2$ & $98.6 \pm 30.6$ & $110.3 \pm 45.3$ & 0.299 \\
\hline $\mathrm{ALT}(\mathrm{U} / \mathrm{L})$ & $53 \pm 40.8$ & $51.1 \pm 25.1$ & $53.2 \pm 42.1$ & 0.837 \\
\hline Number of diseased vessels & $2.6 \pm 0.8$ & $1 \pm 0$ & $2.8 \pm 0.7^{\star \star}$ & 0 \\
\hline \multicolumn{5}{|l|}{ Location lesion [n(\%)] } \\
\hline LM & $28(15.6 \%)$ & $0(0)$ & $28(17.2 \%)$ & 0.063 \\
\hline LAD & $169(93.9 \%)$ & $13(76.5 \%)$ & $156(95.7 \%)^{\star \star}$ & 0.002 \\
\hline LCX & $131(72.8 \%)$ & $4(23.5 \%)$ & $127(77.9 \%)^{\star \star}$ & 0 \\
\hline $\mathrm{RCA}$ & $144(80 \%)$ & $0(0)$ & $144(88.3 \%)^{\star \star}$ & 0 \\
\hline \multicolumn{5}{|l|}{ Laboratory parameters } \\
\hline $\mathrm{TG}\left(\mathrm{mmol} \cdot \mathrm{L}^{-1}\right)$ & $1.92 \pm 1.51$ & $1.88 \pm 1.18$ & $1.93 \pm 1.54$ & 0.876 \\
\hline $\mathrm{TC}\left(\mathrm{mmol} \cdot \mathrm{L}^{-1}\right)$ & $5.19 \pm 1.38$ & $5.55 \pm 1.41$ & $5.16 \pm 1.37$ & 0.268 \\
\hline $\mathrm{LDL}-\mathrm{C}\left(\mathrm{mmol} \cdot \mathrm{L}^{-1}\right)$ & $3.08 \pm 0.97$ & $3.20 \pm 1.01$ & $3.07 \pm 0.97$ & 0.605 \\
\hline $\mathrm{HDL}-\mathrm{C}\left(\mathrm{mmol} \cdot \mathrm{L}^{-1}\right)$ & $1.18 \pm 0.28$ & $1.28 \pm 0.30$ & $1.17 \pm 0.28$ & 0.103 \\
\hline \multicolumn{5}{|c|}{$\begin{array}{l}\text { Data are presented as mean } \pm \text { SD. }{ }^{*} p<0.05,{ }^{* *} p<0.01 \text { (Multivessel Lesions vs Single Lesion). SBP, } \\
\text { systolic blood pressure; DBP, diastolic blood pressure; CREA, creatinine; ALT, alanine transaminase; LM, } \\
\text { left main disease; LAD, left anterior descending artery } \text { LCX, left circumflex; RCA, right coronary artery; TG, } \\
\text { triglyceride; TC, total cholesterol; HDL-C, high density lipoprotein cholesterol; LDL-C, low density } \\
\text { lipoprotein cholesterol. }\end{array}$} \\
\hline
\end{tabular}

\section{Effect of the risk factors for AMI patients with coronary multivessel lesions}

We further analyzed the risk factors of coronary multivessel lesions for all subjects, including age, gender, alcohol, smoking, hypertension, diabetes, ALDH2 mutation, TG, TC, HDL-C, LDL-C. The patients were divided into two groups according to with or without multivessel lesions. The logistics regression analysis showed that age and AIDH2 mutation were more susceptible to coronary multivessel lesions in this ethnic Hakka population with AMl cohort (Table 4). 
Table 4

Multivariate Logistic Regression Analyses

for Predictors of Multivessel Lesions in All

Subjects

\begin{tabular}{|c|c|c|}
\hline Variable & Odds Ratio (95\%Cl) & $P$ value \\
\hline Age & $1.05(1.02-1.08)^{* *}$ & 0.002 \\
\hline Gender & $0.83(0.26-2.67)$ & 0.752 \\
\hline Alcohol & $0.54(0.16-1.78)$ & 0.310 \\
\hline Smoking & $0.46(0.19-1.14)$ & 0.093 \\
\hline Hypertension & $1.33(0.64-2.75)$ & 0.444 \\
\hline Diabetes & $1.34(0.58-3.06)$ & 0.494 \\
\hline ALDH2 mutation & $2.07(1.02-4.22)$ * & 0.045 \\
\hline TTG & $0.83(0.60-1.15)$ & 0.254 \\
\hline TC & $1.00(0.38-2.59)$ & 0.995 \\
\hline LDL-C & $1.33(0.43-4.15)$ & 0.620 \\
\hline HDL-C & $0.27(0.05-1.53)$ & 0.139 \\
\hline${ }^{\star} p<0.05,{ }^{\star \star} p<0$ & 0.01 & \\
\hline
\end{tabular}

\section{Discussion}

In this study, we found that the ethnic Hakka population with AMI carrying mutated ALDH2 allele was associated with increased risk of multiple coronary artery lesions. There is a hypothesis that multiple coronary artery lesions and ALDH2 genotype is closely related to the ethnic Hakka population with AMI. To confirmed the assumption, we analyzed the characteristics of the single lesion group and the multivessel lesions group in ALDH2 wild genotype group or mutated genotype group, respectively. The AMI with ALDH2 wild genotype patients with lower DBP were more likely to be older, have hypertension, elevated diseased vessels in multivessel lesions group. In addition, single lesion group had higher levels of the TG and lower levels of the LDL-C compare to the multivessel lesions group. The AMI with ALDH2 mutated genotype patients also tend to be elderly and increased diseased vessels in multivessel lesions group. The multivessel lesions group had higher levels of the TG and lower levels of the LDL-C than the single lesion group, but it is no statistical differences. The logistics regression showed that age and ALDH2 mutation were vulnerable to the multivessel lesions the ethnic Hakka population with AMI.

ALDH2 is a key enzyme involved in the detoxification of alcohol metabolite acetaldehyde. It is also involved in the oxidation of other aldehydes. It not only has dehydrogenase activity, but also has reductase and esterase activity[19]. The ALDH2 mutant enzyme activity was significantly reduced, and the ALDH2 mutant homozygote basically eliminated its dehydrogenase activity, and the heterozygote was only about $6 \%[20]$. In recent years, it has been shown that ALDH2 may play a role in cardioprotection by oxidative detoxification of aldehydes, etc. Ebert et al.[21] confirmed that ALDH2 mutants increase ROS and toxic aldehydes in human pluripotent stem cell-derived cardiomyocytes, thereby inducing cells cyclic arrest and activation of apoptotic signaling pathways, especially during ischemic injury, reduce myocardial cell survival. When the ALDH2 gene is mutated, it will reduce the activity of ALDH2 and make ethanol in vivo. The procedure of alcohol consumption that ethanol $\rightarrow$ acetaldehyde $\rightarrow$ Water, carbon dioxide metabolism is blocked, a large amount of acetaldehyde will be stayed in the body, will increase the severity of healthy. People with mutation in the ALDH2 gene cannot quickly complete the alcohol metabolism process, resulting in the accumulation of acetaldehyde in the body will cause serious 
damage to the liver, kidney, heart and brain of the human body. In recent years, there have been more and more studies on ALDH2 Glu504Lys polymorphism and cardiovascular disease, especially coronary heart disease. Firstly, Japanese researcher Takagi et al found that the ALDH2 2 allele is an independent risk factor for myocardial infarction in the Japanese population after adjusting for traditional cardiovascular risk factors and alcohol consumption[22]. Subsequently, Hashimoto et al[23] also aimed at Japanese males, suggesting that people with ALDH2 mutations have a higher risk of coronary heart disease. Moreover, Chinese scholar Guo et al.[24] obtained similar conclusions to Takagi and Hashimoto that ALDH2 2 allele is an acute coronary syndrome (ACS) that is independent of traditional risk factors and drinking in the Han population or risk factors for coronary heart disease. More importantly, a Japanese genome-wide association study in 2012 found that ALDH2 Glu504Lys is a genetic susceptibility locus for coronary heart disease[25]. A growing number of evidence suggests that the ALDH2 gene mutation is one of the risk factors for coronary heart disease. Our study also showed that ALDH2 ${ }^{*} 2$ allele is an independent risk factor for myocardial infarction Hakka population with on coronary artery lesions

Multivessel lesions was associated with lower diastolic blood pressure (DBP) in wild genotype patients with AMI $(77.3 \pm 14.1$ versus $86.4 \pm 13.8, p<0.01)[26$, 27]. Our finding was in consisitent with previous analyses lower DBP is an increased risk for cardiovascular events and acute myocardial infarction. Other study has confirmed that ALDH2 wild genotype is a risk factor for hypertension in Asian population (OR = 1.31, 95\% Cl 1.18-1.47, $\mathrm{p}<0.00001$ )[28]. In our research, we found that multivessel lesions group was more likely to have hypertension than single lesion group in ALDH2 wild genotype AMI patients.

With the continuous research of genes, single nucleotide polymorphisms can better explain the differences in individual phenotypes, disease susceptibility, and drug metabolism. The Hakka population is belong to Han Chinese population that have distinct characteristics of lifestyle, eating habits, cultural folklore and other characteristics that are distinct from other ethnic groups[29]. With the deepening of the research on the exact mechanism of ALDH2 gene and coronary angiography in the development of AMI, it will provide new ideas and therapeutic targets for AMI risk prediction and individualized treatment. The ALDH2 gene is only one of them, but the pathogenesis of coronary heart disease is complex, and it is involved by genetic and environmental factors. The mechanism of ALDH2 gene mutation and coronary vascular disease involved in the pathogenesis remains to be further studied. Besides, the study patients are too small, it is vital to expand the sample size to further explore.

\section{Conclusions}

The AMI patients in ethnic Hakka population carrying mutated ALDH2 allele was vulnerable to multiple coronary artery lesions. ALDH2 allele was associated with multiple coronary artery lesions in AMI patients among ethnic Hakka population.

\section{Abbreviations}


ALDH2: Aldehyde dehydrogenase 2; AMI: acute myocardial infarction ; SBP: systolic blood pressure; DBP: diastolic blood pressure; CREA: creatinine ; ALT: alanine transaminase; LM: left main disease; LAD: left anterior descending artery; LCX: left circumflex; RCA: right coronary artery; TG: triglyceride; TC: total cholesterol; HDL-C: high density lipoprotein cholesterol; LDL-C: low density lipoprotein cholesterol.

\section{Declarations}

\section{Ethics approval and consent to participate}

The study was approved by the Ethics Committee of Meizhou People's Hospital, Meizhou Academy of Medical Sciences, Meizhou Hospital Affiliated to Sun Yat-sen University. We obtained individuals signed the written informed consents form before the study.

\section{Consent for publication}

Not applicable.

\section{Conflicts of interest}

There are no conflicts of interest.

\section{Acknowledgements}

The author would like to thank other colleagues whom were not listed in the authorship and for their helpful comments on the manuscript of Research Experimental Center, Meizhou People's Hospital (Huangtang Hospital), Meizhou Hospital Affiliated to Sun Yat-sen University.

\section{Author's contributions}

ZZ designed the study and analyzed the data; CG wrote the manuscript; SL recruited subjects and collected clinical data; XG, RW, ZZ helped to analyze the data; CG conducted the laboratory testing. All authors read and approved the final manuscript.

\section{Funding}

This study was supported by the grants from Science and Technology Program of Meizhou (2019B0202001), Guangdong Provincial Key Laboratory of Precision Medicine and Clinical Translation Research of Hakka Population (2018B030322003), Key Scientific and Technological Project of Meizhou People's Hospital (MPHKSTP-20180101).

\section{Availability of data and materials}

The datasets generated during the current study are available from the corresponding author on reasonable request. 


\section{Author details}

${ }^{1}$ Research Experimental Center, Meizhou People's Hospital (Huangtang Hospital), Meizhou Academy of Medical Sciences, Meizhou Hospital Affiliated to Sun Yat-sen University, No 63 Huangtang Road, Meijiang District, Meizhou 514031, People's Republic of China. ${ }^{2}$ Guangdong Provincial Engineering and Technological Research Center for Molecular Diagnostics of Cardiovascular Diseases, No 63 Huangtang Road, Meijiang District, Meizhou 514031, People's Republic of China. ${ }^{3}$ Provincial Key Laboratory of Precision Medicine and Clinical Translational Research of Hakka Population, No 63 Huangtang Road, Meijiang District, Meizhou 514031, People's Republic of China. ${ }^{4}$ Center for Cardiovascular Diseases, Meizhou People's Hospital (Huangtang Hospital), Meizhou Academy of Medical Sciences, Meizhou Hospital Affiliated to Sun Yat-sen University, Meizhou 514031, People's Republic of China.

\section{References}

1. Anderson JL, Morrow DA. Acute Myocardial Infarction. The New England journal of medicine 2017, 376(21):2053-2064.

2. Writing Group M, Mozaffarian D, Benjamin EJ, Go AS, Arnett DK, Blaha MJ, Cushman M, Das SR, de Ferranti S, Despres JP, et al. Heart Disease and Stroke Statistics-2016 Update: A Report From the American Heart Association. Circulation. 2016;133(4):e38-60.

3. $\mathrm{Li} \mathrm{H}, \mathrm{Ge} \mathrm{J}$. Cardiovascular diseases in China: Current status and future perspectives. International journal of cardiology Heart vasculature. 2015;6:25-31.

4. Nadir MA. Letter by Nadir Regarding Article, "Prevalence, Clinical Features, and Prognosis of Acute Myocardial Infarction Attributable to Coronary Artery Embolism". Circulation. 2016;133(5):e378.

5. Chen CM. [A gold mine of information-the nationwide nutrition and health survey in China, 2002]. Zhonghua liu xing bing xue za zhi = Zhonghua liuxingbingxue zazhi 2005, 26(7):469-470.

6. Li G, de Courten M, Jiao S, Wang Y. Prevalence and characteristics of the metabolic syndrome among adults in Beijing, China. Asia Pac J Clin Nutr. 2010;19(1):98-102.

7. Liu H, Tian Y, Xiang X, Sun K, Juan J, Song J, Cao Y, Xu B, Hu Y. Air Pollution and Hospitalization for Acute Myocardial Infarction in China. The American journal of cardiology. 2017;120(5):753-8.

8. Ma GS, Li YP, Wu YF, Zhai FY, Cui ZH, Hu XQ, Luan DC, Hu YH, Yang XG. [The prevalence of body overweight and obesity and its changes among Chinese people during 1992 to 2002]. Zhonghua yu fang yi xue za zhi [Chinese journal of preventive medicine] 2005, 39(5):311-315.

9. Morrone D, Weintraub WS, Toth PP, Hanson ME, Lowe RS, Lin J, Shah AK, Tershakovec AM. Lipidaltering efficacy of ezetimibe plus statin and statin monotherapy and identification of factors associated with treatment response: a pooled analysis of over 21,000 subjects from 27 clinical trials. Atherosclerosis. 2012;223(2):251-61.

10. Bainey KR, Mehta SR, Lai T, Welsh RC. Complete vs culprit-only revascularization for patients with multivessel disease undergoing primary percutaneous coronary intervention for ST-segment 
elevation myocardial infarction: a systematic review and meta-analysis. American heart journal. 2014;167(1):1-14 e12.

11. Vlaar PJ, Mahmoud KD, Holmes DR Jr, van Valkenhoef G, Hillege HL, van der Horst IC, Zijlstra F, de Smet BJ: Culprit vessel only versus multivessel and staged percutaneous coronary intervention for multivessel disease in patients presenting with ST-segment elevation myocardial infarction: a pairwise and network meta-analysis. Journal of the American College of Cardiology 2011, 58(7):692-703.

12. Park HW, Yoon CH, Kang SH, Choi DJ, Kim HS, Cho MC, Kim YJ, Chae SC, Yoon JH, Gwon HC, et al. Early- and late-term clinical outcome and their predictors in patients with ST-segment elevation myocardial infarction and non-ST-segment elevation myocardial infarction. Int J Cardiol. 2013;169(4):254-61.

13. Toma M, Buller CE, Westerhout CM, Fu Y, O'Neill WW, Holmes DR Jr, Hamm CW, Granger CB, Armstrong PW, Investigators A-A. Non-culprit coronary artery percutaneous coronary intervention during acute ST-segment elevation myocardial infarction: insights from the APEX-AMI trial. European heart journal. 2010;31(14):1701-7.

14. Bosron WF, Lumeng L, Li TK. Genetic polymorphism of enzymes of alcohol metabolism and susceptibility to alcoholic liver disease. Mol Aspects Med. 1988;10(2):147-58.

15. Mackenzie IS, Maki-Petaja KM, McEniery CM, Bao YP, Wallace SM, Cheriyan J, Monteith S, Brown MJ, Wilkinson IB. Aldehyde dehydrogenase 2 plays a role in the bioactivation of nitroglycerin in humans. Arterioscler Thromb Vasc Biol. 2005;25(9):1891-5.

16. Kim JS, Kim YJ, Kim TY, Song JY, Cho YH, Park YC, Chung HW. Association of ALDH2 polymorphism with sensitivity to acetaldehyde-induced micronuclei and facial flushing after alcohol intake. Toxicology. 2005;210(2-3):169-74.

17. Zhang J, Chen Z, Cobb FR, Stamler JS. Role of mitochondrial aldehyde dehydrogenase in nitroglycerin-induced vasodilation of coronary and systemic vessels: an intact canine model. Circulation. 2004;110(6):750-5.

18. Yoshida A, Huang IY, Ikawa M. Molecular abnormality of an inactive aldehyde dehydrogenase variant commonly found in Orientals. Proc Natl Acad Sci USA. 1984;81(1):258-61.

19. Chen CH, Ferreira JC, Gross ER, Mochly-Rosen D. Targeting aldehyde dehydrogenase 2: new therapeutic opportunities. Physiological reviews. 2014;94(1):1-34.

20. Crabb DW, Edenberg HJ, Bosron WF, Li TK. Genotypes for aldehyde dehydrogenase deficiency and alcohol sensitivity. The inactive ALDH2(2) allele is dominant. J Clin Investig. 1989;83(1):314-6.

21. Ebert AD, Kodo K, Liang P, Wu H, Huber BC, Riegler J, Churko J, Lee J, de Almeida P, Lan F, et al. Characterization of the molecular mechanisms underlying increased ischemic damage in the aldehyde dehydrogenase 2 genetic polymorphism using a human induced pluripotent stem cell model system. Science translational medicine. 2014;6(255):255ra130.

22. Takagi S, Iwai N, Yamauchi R, Kojima S, Yasuno S, Baba T, Terashima M, Tsutsumi Y, Suzuki S, Morii I, et al. Aldehyde dehydrogenase 2 gene is a risk factor for myocardial infarction in Japanese men. 
Hypertension research: official journal of the Japanese Society of Hypertension. 2002;25(5):677-81.

23. Hashimoto Y, Nakayama T, Futamura A, Omura M, Nakarai H, Nakahara K. Relationship between genetic polymorphisms of alcohol-metabolizing enzymes and changes in risk factors for coronary heart disease associated with alcohol consumption. Clinical chemistry. 2002;48(7):1043-8.

24. Guo YJ, Chen L, Bai YP, Li L, Sun J, Zhang GG, Yang TL, Xia J, Li YJ, Chen XP. The ALDH2 Glu504Lys polymorphism is associated with coronary artery disease in Han Chinese: Relation with endothelial ADMA levels. Atherosclerosis. 2010;211(2):545-50.

25. Takeuchi F, Yokota M, Yamamoto K, Nakashima E, Katsuya T, Asano H, Isono M, Nabika T, Sugiyama T, Fujioka A, et al. Genome-wide association study of coronary artery disease in the Japanese. European journal of human genetics: EJHG. 2012;20(3):333-40.

26. McEvoy JW, Chen Y, Rawlings A, Hoogeveen RC, Ballantyne CM, Blumenthal RS, Coresh J, Selvin E. Diastolic Blood Pressure, Subclinical Myocardial Damage, and Cardiac Events: Implications for Blood Pressure Control. J Am Coll Cardiol. 2016;68(16):1713-22.

27. Vidal-Petiot E, Greenlaw N, Ford I, Ferrari R, Fox KM, Tardif JC, Tendera M, Parkhomenko A, Bhatt DL, Steg PG. Relationships Between Components of Blood Pressure and Cardiovascular Events in Patients with Stable Coronary Artery Disease and Hypertension. Hypertension. 2018;71(1):168-76.

28. Jia K, Wang H, Dong P: Aldehyde dehydrogenase 2 (ALDH2) Glu504Lys polymorphism is associated with hypertension risk in Asians: a meta-analysis. International journal of clinical and experimental medicine 2015, 8(7):10767-10772.

29. Wang WZ, Wang CY, Cheng YT, Xu AL, Zhu CL, Wu SF, Kong QP, Zhang YP. Tracing the origins of Hakka and Chaoshanese by mitochondrial DNA analysis. Am J Phys Anthropol. 2010;141(1):12430 . 